\title{
Oral Concentrated Grape Juice Suppresses Expression of NF-kappa B, TNF- $\alpha$ and iNOS in Experimentally Induced Colorectal Carcinogenesis in Wistar Rats
}

\author{
Vanessa Maria de Lima Pazine Campanholo, Roseane Mendes Silva, Tiago \\ Donizetti Silva, Ricardo Artigiani Neto, Ana Paula Ribeiro Paiotti, Daniel Araki \\ Ribeiro, Nora Manoukian Forones*
}

\begin{abstract}
The aim of this study was to evaluate the effects of grape juice on colon carcinogenesis induced by azoxymethane (AOM) and expression of NF-kB, iNOS and TNF- $\alpha$. Methods: Forty male Wistar rats were divided into 7 groups: G1, control; G2, 15 mg/kg AOM; G3, $1 \%$ grape juice 2 weeks before AOM; G4, $2 \%$ grape juice 2 weeks before AOM; G5, 1\% grape juice 4 weeks after AOM; G6, 2\% grape juice 4 weeks after AOM; G7, 2\% grape juice without AOM. Histological changes and aberrant crypt foci (ACF) were studied, while RNA expression of NFkB, TNF- and iNOS was evaluated by qPCR. Results: The number of ACF was higher in G2, and G4 presented a smaller number of crypts per focus than G5 (p=0.009) and G6. Small ACF (1-3) were more frequent in G4 compared to $\mathrm{G} 2, \mathrm{G5}$ and $\mathrm{G6}$ ( $\mathrm{p}=0.009, \mathrm{p}=0.009$ and $\mathrm{p}=0.041$, respectively). RNA expression of NF-kB was lower in $\mathrm{G} 3$ and $\mathrm{G} 4$ compared to $\mathrm{G} 2$ ( $\mathrm{p}=\mathbf{0 . 0 0 4}$ and $\mathrm{p}=\mathbf{0 . 0 0 2}$, respectively). A positive correlation was observed between TNF $-\alpha$ and NF-kB gene expression $(p=0.002)$. In conclusion, the administration of $2 \%$ grape juice before AOM reduced the crypt multiplicity, attenuating carcinogenesis. Lower expression of NF-kB was observed in animals exposed to grape juice for a longer period of time, regardless of concentration.
\end{abstract}

Keywords: Grape juice - NF-kappa B - TNF- $\alpha$ - iNOS - carcinogenesis

Asian Pac J Cancer Prev, 16 (3), 947-952

\section{Introduction}

Colorectal cancer shows a high incidence and mortality throughout the world despite advances in the diagnosis and treatment of this cancer. Genetic and environmental factors have been associated with colorectal cancer carcinogenesis (Jemal et al., 2010). The difficulty in detecting this cancer in its early stage due to insidious clinical manifestations has encouraged colorectal cancer prevention campaigns and the identification of foods containing molecules with chemopreventive properties that may inhibit carcinogenesis (Moreira, 2012).

A phytochemical or chemopreventive agent possesses the ability to prevent tumor development. This ability is not the result of a single biological response, but is due to the combination of different intracellular effects (Surh, 2003). Resveratrol has recently been shown to inhibit the three stages of carcinogenesis: initiation, promotion, and progression (Wallerath et al., 2002).

A possible mechanism of action involves the inhibition of iNOS. Wallerath et al. (2002) suggested that resveratrol increased the activity of endothelial NOS (eNOS) promoter in human endothelial cells.

Velmurugan et al. (2010) showed that grape seed extract reduced the formation of aberrant crypt foci induced by azoxymethane (AOM), suppressed the signaling of $\beta$-catenin and $\mathrm{NF}-\mathrm{Kb}$, inhibited cell proliferation and induced apoptosis through reduction of cyclin D1, survivin, iNOS and COX -2 .

AOM is commonly used for the induction of colorectal carcinogenesis in rodents. The model of experimental carcinogenesis induction resembles the characteristics of sporadic colorectal cancer in humans. This fact makes this model an important tool for the study of different aspects of colorectal cancer that cannot be investigated in humans, such as chemopreventive agents and therapeutic strategies (Perse et al., 2011).

Aberrant crypt foci (ACF) are the first manifestation during the development of colorectal cancer, which can be identified microscopically on the surface of the colon mucosa after staining with methylene blue. Studies on rodents and humans, including molecular analysis, have shown that ACF are a valuable intermediate marker of colon carcinogenesis (Bird, 1995). 
The inflammatory process characteristic of colorectal carcinogenesis is promoted by mediators such as tumor necrosis factor alpha (TNF- $\alpha$ ), nuclear transcription factor kappa B (NF-kB), endotoxins, and high expression of adhesion molecules in local endothelial cells. As a consequence, reduced expression of these genes may interfere with carcinogenesis (Terzic et al., 2010). Altered regulation of NF-kB is observed in many cancers. This transcription factor modulates the activation of genes related to cell proliferation, angiogenesis, metastasis, tumor promotion, inflammation, and apoptosis suppression (Watanabe et al., 2000; Lind et al., 2001; Cilloni et al., 2008; Meteoglu et al., 2008). Inhibition of the NF-kB signaling pathway is therefore a promising target for chemopreventive and chemotherapeutic approaches. A diet containing grape seed extract, as well as other polyphenols such as resveratrol, has been shown to suppress NF-kB activity, inhibiting the growth of cancer cells (Watanabe et al., 2000).

Studies suggest that the development of colorectal cancer involves inflammatory mechanisms in which inducible nitric oxide synthase (iNOS) plays a critical role. This enzyme is known to participate in chronic inflammation, creating a microenvironment that favors colon carcinogenesis. Several lines of evidence indicate that iNOS levels are increased in human adenocarcinomas and in colon tumors chemically induced in rats. Therefore, suppression of the synthesis or activity of this enzyme represents a possible target for cancer chemoprevention (Manson, 2003).

The aim of the present study was to evaluate the effects of concentrated grape juice on colon carcinogenesis induced by AOM in Wistar rats. For this purpose, we determined the number of aberrant crypts and RNA expression of NF-kB, iNOS and TNF- $\alpha$.

\section{Materials and Methods}

\section{Animals and treatment protocol}

Forty male Wistar rats (Rattus norvegicus albinus), aged 5 to 6 weeks and weighing 200-280 g, provided by the Center for the Development of Experimental Models in Medicine and Biology (CEDEME), Universidade Federal de São Paulo - Escola Paulista de Medicina (UNIFESPEPM), were used. All procedures were in accordance with guidelines on animal experimentation and the Ethics Committee of UNIFESP-EPM approved the study.

The animals were maintained in plastic cages with metal bars at a temperature of $23^{\circ} \mathrm{C}$ under a $12 \mathrm{~h}$ light/dark cycle for 3 days prior to the experiment for adaptation to the new environment. The animals had free access to water and standard chow.

After one week of adaptation, the animals were randomly divided into seven groups of 5-7 animals each. Animals of groups 2, 3, 4, 5 and 6 received two intraperitoneal injections of $15 \mathrm{mg} / \mathrm{kg} \mathrm{AOM}$ at an interval of one week.

The animals received, daily, by oral gavage $222 \mathrm{mg}$ / day (1\%concentration) or $444 \mathrm{mg} /$ day (2\%concentration) of concentrated grape (G8000) supplied by Gold juice (Farringdon, RS, Brazil). The dose was calculated on the basis of the amount of polyphenols present in four glasses $(200 \mathrm{~mL}$ each) of natural grape juice adjusted to the animal metabolism (twice as fast as humans) (Morris et al., 1989). This dose corresponds to $1 \%$ of concentrated grape juice. The total dose of the juice according weight of rats was $1.18 \mathrm{~g} / \mathrm{kg} /$ day ( $1 \%$ grape juice) or $2.36 \mathrm{~g} / \mathrm{kg} /$ day ( $2 \%$ grape juice). The chemical characterization of grape juice concentrate had previously described in a study conducted by our research group (Hasler et al., 2004).

Groups 1 and 2 received water and the remaining groups received 1\% (groups 3 and 5) or 2\% (groups 4 and 6) grape juice by gavage. Grape juice was administered two weeks before the first application of AOM in groups 3 and 4 , and 4 weeks after the first application of AOM in groups 5 and 6 . Animals of group 7 received $2 \%$ grape juice throughout the experiment. The animals were sacrified after 22 weeks (Figure 1).

\section{Analysis of aberrant crypt foci}

After surgery, colon fragments (approximately $1 \mathrm{~cm}$ ) were collected from each animal for RNA extraction. The colon of each animal was stretched out, mounted on a Styrofoam board, and fixed in $10 \%$ buffered formalin for $24 \mathrm{~h}$. Next, the specimens were washed in water for $12 \mathrm{~h}$ and stored in $70 \%$ alcohol until the time for analysis. For the analysis of ACF, the colons were removed from $70 \%$ alcohol, washed under running water, and stained with $0.2 \%$ methylene blue in water for approximately 2 min. The colon was cut into distal, medial and proximal portions and each was mounted on a slide and analyzed under a light microscope at 40x magnification.

ACF were identified and quantified based on their morphological features according to Bird (1995). The number of ACF was divided in 3 groups 1-3, 4-9 or $>10$. The total number of aberrant crypts was also calculated per colon segment. The specimens were always analyzed in the distal to proximal direction.

The colon portions were cut longitudinally to provide a perpendicular view of the crypts and transferred to plastic cassettes. All fragments were processed for histological analysis and stained with hematoxylin-eosin.

\section{Extraction of total RNA}

Frozen tissue was homogenized and total RNA was

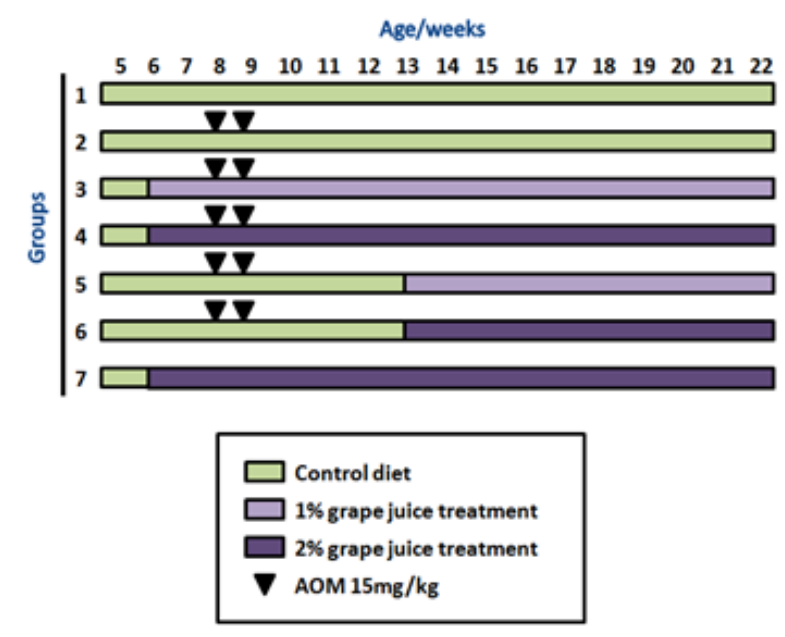

Figure 1. Experimental Design 
extracted with cold Trizol reagent (Invitrogen, Carlsbad, CA, USA) according to manufacturer instructions. RNA integrity and purity were analyzed in a NanoDrop ${ }^{\circledR}$ ND-1000 spectrophotometer (NanoDrop Technologies, Wilmington, DE, USA). The RNA samples were treated with DNase (Invitrogen) to prevent contamination with genomic DNA.

\section{Synthesis of complementary DNA and real-time PCR}

cDNA was synthesized using M-MVL reverse transcriptase (Invitrogen) according to manufacturer instruction. Pre-designed TaqMan Gene Expression Assays were used for the five genes studied (TNF- $\alpha$ : Rn00562055_m1; NF-kB: Rn01399583_m1; iNOS: Rn00561646_m1; GAPDH: Rn01775763_g1; $\beta$-actin: Rn00667869_m1). Each reaction was carried in triplicate in a final volume of $20 \mu \mathrm{L}$. Positive and negative controls were included in each assay. The reaction mixture contained $10 \mu \mathrm{L}$ TaqMan Gene Expression Mix (Applied Biosystems, UK), $1 \mu \mathrm{L}$ TaqMan Gene Expression Assay. (20x) (Applied Biosystems), $2 \mu \mathrm{L}$ cDNA, and $7 \mu \mathrm{L}$ nuclease-free water. The reaction was carried out in a Step One Plus thermocycler (Applied Biosystem) under the following conditions: incubation at $50 \mathrm{oC}$ for $2 \mathrm{~min}$ and at $95 \mathrm{oC}$ for $10 \mathrm{~min}$, followed by 40 cycles at $95 \mathrm{oC}$ for $15 \mathrm{~s}$ and at $60 \mathrm{oC}$ for $1 \mathrm{~min}$.

\section{Statistical analysis}

The values are presented by median and quartile range. The Kruskal-Wallis test was used to determine differences in weight, ACF number and mRNA expression of the genes studied. If a difference was detected, pairwise comparison was performed by the Mann-Whitney test. Pearson's correlation coefficient was used to evaluate the association between two continuous variables. Statistical analysis was performed using the SPSS 10.01 (SPSS, Inc.) and Statistica 7 (Statsoft, Inc.) programs. A level of significance of $0.05(\alpha=5.0 \%)$ was adopted and a $P$ value $<0.05$ was considered to be significant.

\section{Results}

\section{Clinical assessment and macroscopic analysis}

No significant difference in body weight was observed between groups. There was no difference in water or food intake, and none of the animals presented pain or discomfort. The animals were clinically healthy and no death occurred during the experiment. During surgery, no macroscopic alterations were seen in the liver, stomach, kidneys, or heart. The colons of animals submitted to AOM-mediated tumor induction exhibited mild hyperemia when compared to the groups not submitted to such induction.

\section{Histopathological findings}

A normal colon wall showing no signs of histopathological alterations was observed in the control group (Figure 2A). Animals that only received an intraperitoneal injection of AOM presented increased inflammatory infiltration of the chorion accompanied by proliferative epithelial alterations, diffuse glandular hyperplasia, and aberrant crypts lined with cells containing voluminous nuclei compared (Figure 2B). Attenuation of these intestinal alterations was observed in the groups receiving $1 \%$ and $2 \%$ grape juice through a feeding tube before and after AOM application. However, the changes were still evident when compared to the control groups (Figure 2C, D, E and F).

\section{Number of aberrant crypt foci}

The number of ACF and the total number of aberrant crypts per ACF per colon segment had been calculated and demonstrated in Table 1 for each group. No aberrant crypts were detected in animals not treated with AOM (groups 1 and 7). ACF were identified in the groups submitted to AOM-mediated tumor induction (groups 2 to 6) and classified according to the number of focus per crypt: $1-3,4-9$, and $\geq 10$.

The groups receiving grape juice and AOM still presented intestinal mucosal alterations, but aberrant crypts were less frequent and less intense. Thus, three groups of animals could be distinguished: control (sham or grape juice), group treated with AOM, and groups receiving AOM and grape juice.

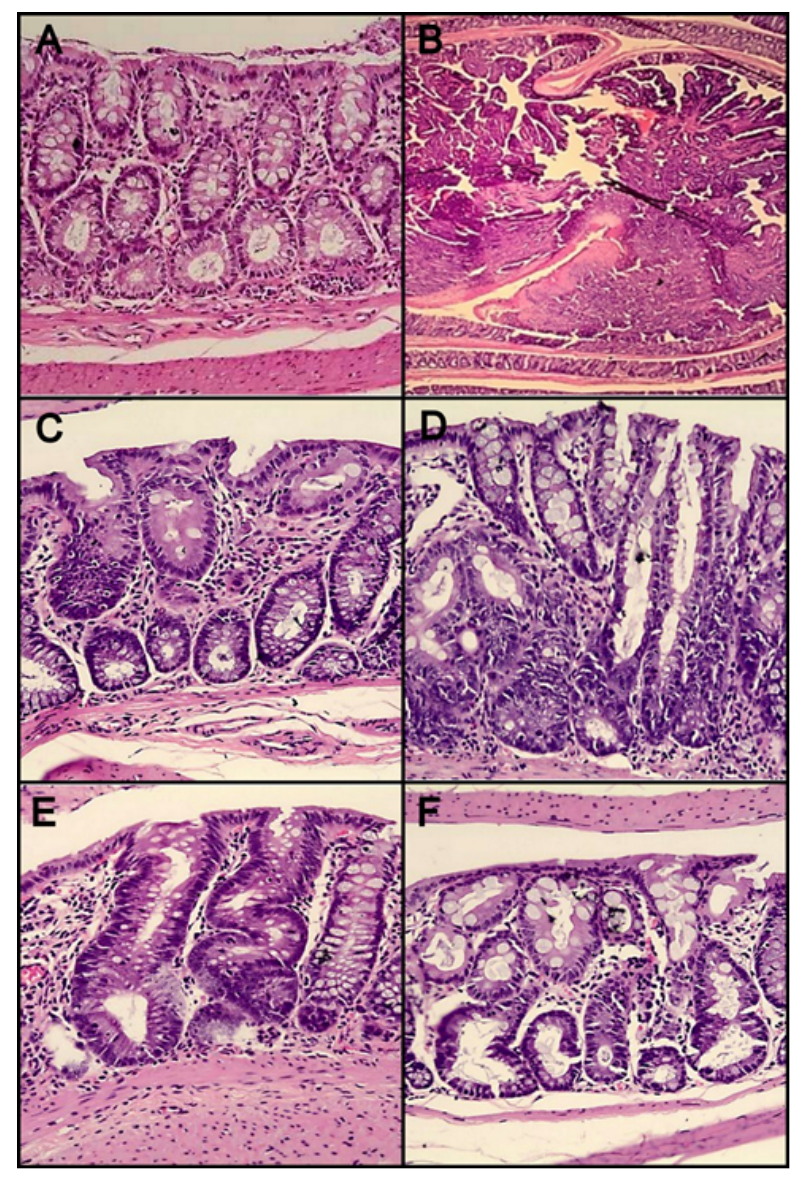

Figure 2. Histopathologic Aspects after Hematoxylineosin Staining. A: control group; B: histopathologic alterations in rat colon after Azoxymethane (AOM). Histopathologic alterations in rat colon of groups submitted to grape juice intake; C: before and after AOM, $1 \%$ grape juice intake before and after AOM; D: $1 \%$ grape juice intake after AOM, E: $2 \%$ grape juice intake before and after AOM; F: $2 \%$ grape juice intake after $\mathrm{AOM}$ 
Table 1. Treatment Effect of Concentrated Grape Juice on Number And Multiplicity of Aberrant Crypt Focus

\begin{tabular}{lccccccc}
\hline Groups & N & AC Total & ACF total & ACF 1-3 & ACF 4-9 & ACF $\geq 10$ & AC/ACF \\
\hline (G2) AOM & 5 & $590.1 \pm 230.1$ & $73.7 \pm 29.4$ & $22.6 \pm 7.7^{\mathrm{a}}$ & $36.3 \pm 14.5$ & $18.9 \pm 9.2$ & $7.7 \pm 0.8^{\mathrm{e}}$ \\
(G3) 1\% before AOM & 6 & $482.7 \pm 234.6$ & $79.4 \pm 38.7$ & $29.1 \pm 20.0$ & $35.5 \pm 16.9$ & $17.2 \pm 8.9$ & $6.3 \pm 1.1$ \\
(G4) 2\%before AOM & 6 & $415.5 \pm 224.8$ & $112.9 \pm 37.3$ & $61.2 \pm 17.6^{\mathrm{b}}$ & $34.0 \pm 7.20$ & $8.0 \pm 12.4$ & $3.9 \pm 1.0^{\mathrm{f}}$ \\
(G5) 1\% after AOM & 6 & $417.7 \pm 126.9$ & $71.4 \pm 25.0$ & $22.0 \pm 11.3^{\mathrm{c}}$ & $30.4 \pm 10.0$ & $14.4 \pm 6.8$ & $7.0 \pm 1.2^{\mathrm{g}}$ \\
(G6) 2\% after AOM & 6 & $488.4 \pm 125.9$ & $77.9 \pm 17.8$ & $27.9 \pm 12.0^{\mathrm{d}}$ & $32.5 \pm 10.9$ & $13.4 \pm 8.8$ & $5.8 \pm 1.6^{\mathrm{h}}$ \\
(G7) 2\% juice & 5 & 0 & 0 & 0 & 0 & 0 & 0 \\
\hline
\end{tabular}

${ }^{* 1}$ values represented by median and quartil range; Kruskal-Wallis test, $a \neq b, p=0.009 ; b \neq c, p=0.009 ; c \neq d, p=0.04 ; e \neq f, p=0.004, f \neq g, p=0.009, f \neq$ h, $\mathrm{p}=0.026$; AOM: azoximethane

Table 2. RNA Gene Expression of NF-KB, TNF- $\alpha$ and iNOS among the Groups

\begin{tabular}{lcccc}
\hline Groups & N & NF-Kb & TNF- $\alpha$ & INOS \\
\hline (G2) AOM & 5 & $0.85 \pm 0.64^{\mathrm{a}}$ & $0.16 \pm 0.57$ & $0.55 \pm 0.78$ \\
(G3) 1\% before AOM & 6 & $0.46 \pm 0.02^{\mathrm{b}}$ & $0.20 \pm 0.13$ & $0.21 \pm 0.10$ \\
(G4) 2\%before AOM & 6 & $0.19 \pm 0.07^{\mathrm{c}}$ & $0.09 \pm 0.05$ & $0.70 \pm 0.18$ \\
(G5) $1 \%$ after AOM & 6 & $0.63 \pm 0.47$ & $0.26 \pm 0.21$ & $0.93 \pm 0.63$ \\
(G6) 2\% after AOM & 6 & $0.31 \pm 0.38$ & $0.00 \pm 0.09$ & $0.94 \pm 0.72$ \\
(G7) 2\% juice & 5 & $0.47 \pm 0.30$ & $0.32 \pm 0.10$ & $0.65 \pm 0.52$
\end{tabular}

${ }^{1}$ Values represented by median and quartile range; $\mathrm{N}=$ number of animals; Kruskal-Wallys test; $a \neq c, p=0.004 ; b \neq c, p=0.002$

Table 3. Pearson Correlation between the Different Genes Studied

\begin{tabular}{lcclc}
\hline & & INOS & NF-kB & TNF-a \\
\hline INOS & correlation & 1 & 0.16 & -0.15 \\
& $\mathrm{p}$ & & 0.33 & 0.45 \\
NF-kB & correlation & 0.16 & 1 & 0.57 \\
& $\mathrm{p}$ & 0.33 & & 0.00 \\
TNF- $\alpha$ & correlation & -0.15 & 0.57 & 1 \\
& $\mathrm{p}$ & 0.45 & 0 & \\
\hline
\end{tabular}

Analysis of $m R N A$ gene expression of $N F-k B, T N F$ - and iNOS

Analysis revealed significant differences in the mRNA expression of NF-kB between groups. In contrast, expression of TNF- $\alpha$ or iNOS did not differ significantly ( $p=0.333$ and $p=0.365$, respectively) (Table 2).

\section{Correlation between iNOS, NF- $k B$ and TNF-gene expression}

The expression of NF-kB and TNF- $\alpha$ was significantly correlated $(\mathrm{p}=0.002)$. However, no significant correlation was observed between the expression of iNOS and NF-kB or TNF- $\alpha$ (Table 3 ).

\section{Discussion}

Chemoprevention is defined as the use of dietary, natural or synthetic compounds to inhibit or reverse the premalignant phase (initiation and promotion) of carcinogenesis. In addition, these substances are able to prevent or delay the development and progression of modified cells in malignant tumors (Marshall, 2008). Preventive approaches such as dietary modulation and chemoprevention can be used successfully to treat colorectal cancer. Epidemiological and experimental evidence indicates that diets rich in fruits and vegetables reduce the risk of gastrointestinal diseases, including colorectal cancer (Pan et al., 2011). Recently, there has been growing interest in the identification of fruit and vegetable compounds and their molecular mechanisms of action in order to elucidate the mechanisms underlying the suppression of colorectal carcinogenesis (Aguiar et al., 2011). Resveratrol is a component found in the concentrated grape juice used in the present study and this compound has been shown to inhibit the three stages of carcinogenesis (Aggarwal et al., 2004).

Aberrant crypt foci are pre-cancerous lesions that can be induced by AOM in the colon of rats and mice. AOM-induced ACF is a useful biomarker of colorectal carcinogenesis and is used to study the anticarcinogenic effects of natural substances (Bird, 1995). In the present study, animals receiving AOM to induce ACF showed no clinical signs of disease or changes in dietary habits, with these animals presenting weight gain proportional to age. It is well established that colorectal cancer in humans is asymptomatic at an early stage and symptoms can be nonspecific even in more advanced disease (Hamilton et al., 2009), a characteristic observed in the present animals.

Concentrated grape juice was administered by gavage before the initiation of carcinogenesis and after the intraperitoneal injection of a carcinogenic drug. Analysis of the number of ACF revealed differences depending on the concentration of the juice and time of exposure, with the observation of a marked reduction of crypt multiplicity in animals of group 4 that received $2 \%$ grape juice before AOM induction compared to the group 2 that was treated only with AOM $(\mathrm{p}=0.004)$. A reduction of crypt multiplicity was also observed in group 4 when animals receiving $1 \%$ or $2 \%$ grape juice after $\mathrm{AOM}$ had been compared (groups 5 and 6$)(\mathrm{p}=0.009$ and $\mathrm{p}=0.026$, respectively).

The number of small ACF (1-3) was higher in group 4 compared to the other groups. This finding suggests that the administration of $2 \%$ grape juice before induction does not inhibit the formation of ACF, but prevents progression to larger crypts, thus affecting multiplicity. Studies have demonstrated the importance of multiplicity of ACF since this parameter indicates a tendency to progress to malignant tumors (Alrawi et al., 2006; Yasui et al., 2008). In agreement with other studies of chemopreventive agents (Murakami, 2009), 2\% grape juice administered before induction by the carcinogen may prevent the progression of these foci to cancer.

In the present study, the administration of concentrated grape juice by gavage caused no apparent sign of toxicity in the animals, which maintained their feeding habits and weight gain pattern, suggesting that constant ingestion of $2 \%$ grape juice is not harmful to the organism. Velmurugan et al (2010), studying the chemopreventive effects of grape 
seed extract, also found no toxicity.

Inducible nitric oxide synthase has been shown to be involved in chronic inflammation, creating a microenvironment that favors colon carcinogenesis. Consequently, suppression of the synthesis or activity of this enzyme is a possible target for cancer chemoprevention. Overexpression of iNOS can cause DNA damage and can increase cell proliferation, resistance to apoptosis, vascularization, and the metastatic potential of the tumor. Therefore, the reduction of iNOS expression may have importance for the prevention and treatment of inflammation-associated carcinogenesis (Li et al., 2012). However, we found no significant difference in iNOS expression levels between groups despite the inhibitory effect of $2 \%$ grape juice administered before induction by AOM on the multiplicity of ACF. These results agree with other studies that were unable to detect a reduction of iNOS expression after treatment with different chemopreventive agents (Baldwin et al., 2001; Aviello et al., 2012; Arulselvan et al., 2012).

$\mathrm{NF}-\mathrm{kB}$ is a transcription factor that regulates cellular processes involved in immediate immune and inflammatory responses, apoptosis and cell proliferation by inducing a variety of target genes (Olivier et al., 2006). In this respect, NF-kB has been shown to play a role in tumorigenesis by activating proliferative and antiapoptotic processes in cancer cells (Popinova et al., 2008).

The present results showed a marked reduction of $\mathrm{NF}-\mathrm{kB}$ expression in animals receiving $2 \%$ grape juice before induction by AOM (group 4) when compared to the group treated only with AOM (group 2) $(\mathrm{p}=0.004)$. The same was observed for group 3 which received $1 \%$ grape juice before induction by AOM ( $\mathrm{p}=0.002)$. The present results indicate that the reduction of focus multiplicity in crypts observed in group 4 might be attributed to the reduced expression of NF-kB, among other factors. The administration of grape juice before induction by AOM may have contributed to its anticarcinogenic effect on AOM-induced ACF formation in rat colon.

It is well established that colorectal cancer is directly related to inflammation. TNF- $\alpha$ is a proinflammatory cytokine that plays a role in the development of colorectal cancer, promoting tumor growth, survival and angiogenesis. In addition, this cytokine is responsible for signal transduction to pre-neoplastic epithelial cells in the colon (Cilloni et al., 2008). Popivanova et al (2008) showed that the inhibition of TNF- $\alpha$ reduces tumorigenesis in rats with cancer induced by AOM and dextran sulfate sodium (DSS). In the study of Li et al (25), colorectal cancer was induced in mice by AOM/DSS and the animals were treated with the chemopreventive agents S-adenosylmethionine and its metabolite methylthioadenosine. As observed in the present study, treatment attenuated tumor multiplicity in the colon, but analysis of TNF- $\alpha$ and iNOS gene expression by real-time PCR revealed no significant differences. Taken together, these findings suggest that the reduction of crypt multiplicity observed in group 4 occurred independently of the expression of TNF- $\alpha$ and iNOS.

Analysis of the expression of NF-kB, TNF- $\alpha$ and iNOS showed increased expression of these genes in the groups with AOM-induced ACF. These findings agree with the literature reporting increased expression of these genes in animals treated with the chemical carcinogen $\mathrm{DMH}$ (Femia et al., 2008). NF-kB and TNF- $\alpha$ participate in the same signaling pathway, a fact suggesting a positive correlation. Studies have shown that the expression of TNF- $\alpha$ depends on the activation of NF-kB, which contributes to inflammation (Azzolina et al., 2003), thus establishing a relationship that may amplify the inflammatory response and prolong the duration of chronic inflammation. The inhibition of NF-kB may therefore be an efficient approach to the prevention of colorectal cancer.

The present results suggest that the inhibition of NF-kB activity with concentrated grape juice modulates inflammatory processes and is positively correlated with the expression of TNF- $\alpha$, in agreement with Umesalma \& Sudhandiran (2010). Others researchers induced hepatocellular carcinoma in knockout rats to study the signal transduction pathways that are activated during early inflammation until advanced disease (carcinoma with metastasis) (Block et al., 2003). Using this model, Pikarsky et al (2004) observed that TNF- $\alpha$ plays a central role in the activation of NF-kB.

In conclusion, $2 \%$ grape juice administered before induction by AOM did not interfere with the formation of $\mathrm{ACF}$, but reduced crypt multiplicity, attenuating colorectal carcinogenesis. Expression of NF-kB was reduced in animals receiving grape juice for a longer period of time, irrespective of its concentration ( $1 \%$ or $2 \%)$. Inhibition of the NF-kB signaling pathway may be one mechanism whereby chemopreventive and chemotherapeutic agents such as concentrated grape juice interfere with colon carcinogenesis.

\section{Acknowledgements}

This work was supported by the Sao Paulo Research Foundation (FAPESP).

\section{References}

Aguiar O Jr, Gollücke AP, de Moraes BB, et al (2011). Grape juice concentrate prevents oxidative DNA damage in peripheral blood cells of rats subjected to a high-cholesterol diet. Br J Nutr, 105, 694-702.

Aggarwal BB, Bhardwaj A, Aggarwal RS, et al (2004). Role of resveratrol in prevention and therapy of cancer: preclinical and clinical studies. Anticancer Res, 24, 2783-840.

Alrawi SJ, Schiff M, Carroll RE, et al (2006). Aberrant crypt foci. Anticancer Res, 26, 107-19.

Arulselvan P, Wen CC, Lan CW, et al (2012). Dietary administration of scallion extract effectively inhibits colorectal tumor growth: cellular and molecular mechanisms in mice. PLoS One, 7, 44658.

Aviello G, Romano B, Borrelli F, et al (2012). Chemopreventive effect of the non-psychotropic phytocannabinoid cannabidiol on experimental colon cancer. J Mol Med, 90, 925-34.

Azzolina A, Bongiovanni A, Lampiasi N (2003). Substance P induces TNF-alpha and IL-6 production through NF kappa B in peritoneal mast cells. Biochim Biophys Acta, 1643, 75-83.

Baldwin AS (2001). Control of carcinogenesis and cancer therapy resistance by the transcription factor NF-Kappa B. 
Vanessa Maria de Lima Pazine Campanholo et al J Clin Invest, 107, 241-246.

Bird RP (1995). Role of aberrant crypt foci in understanding the pathogenesis of colon cancer. Cancer Lett, 93, 55-71.

Block TM, Mehta AS, Fimmel CJ, Jordan R (2003) Molecular viral oncology of hepatocellular carcinoma. Oncogene, 22, 5093-107.

Cilloni D, Messa F, Rosso V, et al (2008). Increase sensitivity to chemotherapeutical agents and cytoplasmatic interaction between NPM leukemic mutant and NF-kappaB in AML carrying NPM1 mutations. Leukemia, 22, 1234-40.

Femia AM, Caderni G (2008). Rodent models of colon carcinogenesis for the study of chemopreventive activity of natural products. Planta Med, 74, 1602-07.

Hamilton W, Lancashire R, Sharp D, et al (2009). The risk of colorectal cancer with symptoms at different ages and between the sexes: a case-control study. BMC Med, 7, 17.

Hasler CM, Bloch AS, Thomson CA, Enrione E, Manning C (2004). Position of the American Dietetic Association:functional foods. J Am Diet Assoc, 5, 814-26.

Jemal A, Siegel R, Xu J, et al (2010). Cancer statistics. Cancer J Clin, 60, 277-300.

Li TW, Yang H, Peng H, et al (2012). Effects of S-adenosylmethionine and methylthioadenosine on inflammation-induced colon cancer in mice. Carcinogenesis, 33, 427-35.

Lind DS, Hochwald SN, Malaty J, et al (2001). NF-kB is upregulated in colorectal cancer. Surgery, 130, 363-9.

Marshall JR (2008). Prevention of colorectal cancer: diet, chemoprevention and lifestyle. Gastroenterol Clin North Am, 37, 73-81

Manson MM (2003). Cancer prevention-the potential for diet to modulate molecular signalling. Trends $\mathrm{Mol} \mathrm{Med,9,11-8.}$

Meteoglu I, Erdogdu IH, Meydan N, Erkus M, Barutca S (2008). NF-KappaB expression correlates with apoptosis and angiogenesis in clear cell renal cell carcinoma tissues. $J$ Exp Clin Cancer Res, 27, 53.

Moreira L (2012). Update on colorectal cancer precursor lesions. Gastroenterol Hepatol, 1, 80-5.

Morris GP, Beck PL, Herridge MS, et al (1989). Hapten-induced model of chronic inflammation and ulceration in the rat colon. Gastroenterology, 96, 795-803.

Murakami A (2009). Chemoprevention with phytochemicals targeting inducible nitric oxide synthase. Forum Nutr, 61, 193-203.

Olivier S, Robe P, Bours V (2006). Can NF-kappaB be a target for novel and efficient anti-cancer agents? Biochem Pharmacol, 72, 1054-68.

Perse M, Cerar A (2011). Morphological and molecular alterations in 1,2 dimethylhydrazine and azoxymethane induced colon. J Biomed Biotechnol, 2011, 473964

Pikarsky E, Porat RM, Stein I, et al (2004). NF-kappaB functions as a tumour promoter in inflammation-associated cancer. Nature, 431, 461-6.

Popivanova BK, Kitamura K, Wu Y, et al (2008). Blocking TNFalpha in mice reduces colorectal carcinogenesis associated with chronic colitis. J Clin Invest, 118, 560-70.

Surh YJ. 2003. Cancer chemoprevention with dietary phytochemicals. Nat Rev Cancer, 3, 768-80

Terzic J, Grivennikov S, Karin E, Karin M (2014). Inflammation and colon cancer. Gastroenterology, 138, 2101-14.

Umesalma S, Sudhandiran G (2010). Differential inhibitory effects of the polyphenol ellagic acid on inflammatory mediators NF-kappaB, iNOS, COX-2, TNF-alpha, and IL-6 in 1,2-dimethylhydrazine-induced rat colon carcinogenesis. Basic Basic Clin Pharmacol Toxicol, 107, 650-5.

Velmurugan B, Singh RP, Agarwal R, Agarwal C (2010). Dietaryfeeding of grape seed extract prevents azoxymethane- induced colonic aberrant crypt foci formation in fischer rats. Mol Carcinog, 49, 641-52.

Wallerath T, Deckert G, Ternes T, et al (2002). Resveratrol, a polyphenolic phytoalexin present in red wine, enhances expression and activity of endothelial nitric oxide synthase. Circulation, 106, 1652-8.

Watanabe K, Kawamori T, Nakatsugi S, Wakabayashi K. (2000) COX-2 and iNOS, good targets for chemoprevention of colon cancer. Biofactors, 12, 129-33.

Yasui Y, Miyamoto S, Kim M, et al (2008). Aqueous and ethanolic extract fractions from the Brazilian propolis suppress azoxymethane-induced aberrant crypt foci in rats. Oncol Rep, 20, 493-9. 